\title{
Targeting lung DCs to treat asthma
}

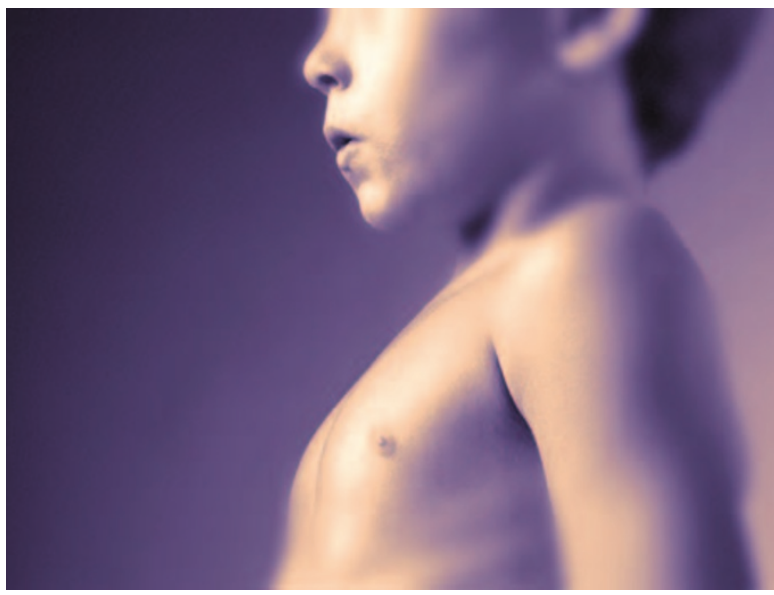

Lung dendritic cells (DCs) are known to have a crucial role in the development of allergic asthma, through

DOI:

10.1038/nri1987 the lymphoid organs, thereby inhibiting lymphocyte influx into sites of inflammation. However, systemic treatment with this agent can result in peripheral lymphopaenia. So, the authors examined the effect of local administration of FTY720 in a mouse model of asthma.

Intratracheal administration of FTY720 before or during allergen challenge of sensitized mice suppressed the cardinal features of asthma, including eosinophilic inflammation, $\mathrm{T}_{\mathrm{H}}$ 2-type cytokine production and bronchial hyperreactivity to a nonspecific stimulus. Notably, the number of lymphocytes in the peripheral blood and lymph nodes was not affected, indicating that lymphopaenia was not induced.

But if FTY720 does not sequester cell responses. Therefore, strategies to interfere with their function might be a novel therapeutic approach for the treatment of asthma. In this study, Lambrecht and colleagues show that local treatment with the immunomodulator FTY720 suppresses experimentally induced asthma through the alteration of lung DC function.

FTY720 is a sphingosine-1-phosphate receptor agonist and is thought to act as an immunosuppressive agent by retaining lymphocytes in the number of DCs in the periphery. However, the number of DCs in the draining lymph nodes of the lung was reduced, whereas the number of DCs detected in the lungs was greatly increased. Therefore, the data indicate that FTY720 prevents the migration of lung DCs to the draining lymph nodes, where they induce allergen-specific $\mathrm{T}_{\mathrm{H}}$ 2-cell responses.

Further examination of the effects of FTY720 on DC function in vitro showed that treatment of DCs with FTY720 directly inhibited the capacity of these cells to activate and polarize antigen-specific T cells. However, the maturation status of the DCs was not affected. The authors showed that the ability of DCs to form stable and long-lasting interactions with $\mathrm{T}$ cells was abolished in the presence of FTY720, which might account for the inability of FTY720-treated DCs to stimulate T cells.

Therefore, FTY720 suppresses experimental asthma by blocking the migration of DCs from the lung to the draining lymph node without inducing peripheral lymphopaenia and by reducing the ability of DCs to activate naive and effector $\mathrm{T}_{\mathrm{H}} 2$ cells. This study indicates that targeting lung DCs might be an effective strategy in the treatment of asthma.

Olive Leavy
ORIGINAL RESEARCH PAPER Idzko, M. et al. Local application of FTY720 to the lung abrogates experimental asthma by altering dendritic cell function. J. Clin. Invest. 116, 2935-2944 (2006) 